\title{
Value of the regurgitant volume to end diastolic volume ratio to predict the regression of left ventricular dimensions after valve replacement in aortic insufficiency
}

\author{
P. Fioretti, J. Roelandt, C. Tirtaman, E. Bos and P. W. Serruys \\ Thoraxcentrum, Erasmus University, Rotterdam and Interuniversity Cardiology Institute, Utrecht, \\ The Netherlands
}

KEY WORDS: valve replacement, aortic insufficiency.

The aim of this study was to assess the value of regurgitant stroke volume ( $R S V$ ) to end-diastolic volume (EDV) ratio to predict the regression of left ventricular $(L V)$ dimensions after uncomplicated valve replacement in 34 patients with severe pure aortic insufficiency. The RSV/EDV ratio was measured by contrast ventriculography and thermodilution techniques. $L V$ end-diastolic diameter (EDD) was measured pre-and postoperatively by $M$ mode echocardiography (at a median interval of $3 \cdot 3$ years after valve replacement). $L V / E D D$ decreased from $74 \pm 8 \mathrm{~mm}$ to $54 \pm 11 \mathrm{~mm}(P<0.001)$.

Eleven patients had a persistent postoperative LV enlargement (median EDD $65 \mathrm{~mm}$, range $56-100 \mathrm{~mm}$ ) while, in 23 patients, EDD became normal (median $49 \mathrm{~mm}$, range $40-55 \mathrm{~mm}$ ). During follow-up, one patient with $L V$ enlargement died of congestive heart failure. Preoperative RSV/EDV ratio was significantly higher in patients with normal postoperative EDD as compared to those with persistent LV enlargement $(0 \cdot 32 \pm 0 \cdot 06$ vs. $0.24 \pm 0.07, P<0.005)$. The best cutoff point of $R S V / E D V$ to predict the normalization of $L V$ dimensions was $0 \cdot 28$. Postoperative EDD remained abnormal in eight out of 16 patients $(50 \%)$ with $R S V / E D V$ ratio less than $0 \cdot 29$, while it remained enlarged in only three out of 18 patients $(17 \%)$ with a preoperative $R V / E D V$ ratio greater than $0 \cdot 28$. The other usual preoperative catheterization and echocardiographic variables were equally or less predictive than RSV/EDV ratio. In conclusion, despite the limitations due to the use of different techniques, we confirmed that the RSV/EDV ratio is a potentially useful variable for the assessment of the proper timing of valve replacement in patients with severe isolated aortic insufficiency.

\section{Introduction}

It has been reported that pre-operative left ventricular dysfunction in severe aortic insufficiency is followed by a poor clinical outcome. ${ }^{[1]}$ However, left ventricular dysfunction does not preclude a good clinical course and a normalisation of left ventricular dimensions in many patients. ${ }^{[2-4]}$

Patients with severe aortic insufficiency and normal left ventricular function have an excellent natural history ${ }^{[5]}$ and, therefore, it is universally agreed that there is no indication for surgery at this stage in absence of symptoms. In contrast, the timing of surgery is uncertain in asymptomatic or oligosymptomatic patients with initial left ventricular dysfunction. ${ }^{[6]}$ Furthermore, at present there is no

Address for correspondence: P. Fioretti, M.D., Thoraxcentrum, P.O. Box 1738, 3000 DR Rotterdam. The Netherlands. accepted single parameter derived from invasive or non-invasive methods to predict with adequate accuracy the clinical course or the outcome of left ventricular function after surgery. ${ }^{[2]}$

Recently, Levine and Gaasch provided some evidence that the relative size of the regurgitant stroke volume and left ventricular end-diastolic volume (RSV/EDV ratio) is a major determinant of the ventricular response to surgical correction of chronic aortic insufficiency. ${ }^{[7]}$

Hence, we retrospectively analyzed the value of the RSV/EDV ratio to predict the normalisation of left ventricular dimensions in 34 patients with pure chronic aortic insufficiency who underwent an uncomplicated valve replacement. We also compared the predictive accuracy of RSV/EDV ratio to that of some routinely used variables derived from M-mode echocardiography and cardiac catheterization. 


\section{Patients and methods}

From 1971 to 1984, all patients with pure chronic aortic insufficiency who underwent cardiac catheterization and subsequently valvular replacement at the Thoraxcenter were considered for this study. Thirty-four patients were included, who fulfilled the following criteria: (1) good quality preand postoperative M-rnode echocardiography; (2) good quality preoperative left ventricular cineventriculogram (right anterior oblique projection), immediately preceded by cardiac output measurement by thermodilution technique; (3) only patients with a difference of heart rate of 15 beats $\mathrm{min}^{-1}$ or less between thermodilution and ventriculography were included in the study.

Left ventricular volumes were measured according to the area-length method. The regression equation used to estimate true volume from the singleplane left ventriculogram is: volume $_{\text {true }}=0.928$ volume $_{\text {measured }}-3.8 \mathrm{ml}^{[8]}$ End-diastolic volume was measured at the peak of the $\mathrm{R}$ wave, and was identified by a system of frame markers recorded simultaneously with the electrocardiogram. Endsystolic volume (ESV) was defined as the smallest left ventricular volume. The following calculations were also performed:

(1) Regurgitant stroke volume index:

$$
\text { RSVI }=\text { ASVI }- \text { TSVI, }
$$

(2) Regurgitant fraction (RF, \%):

$$
\frac{\text { ASVI }- \text { TSVI }}{\text { ASVI }} \times 100,
$$

where ASVI is angiographic stroke volume index and TSVI is thermodilution stroke volume index.

Pre- and postoperative left ventricular end diastolic diameter (EDD), end systolic diameter (ESD), fractional shortening (FS), cross sectional area (CSA) and radius to thickness ratio $(\mathrm{R} / \mathrm{Th})$ were also measured by $\mathrm{M}$-mode echocardiography according to classical criteria. ${ }^{[4]}$ Postoperative echocardiograms were performed at a median interval of $3 \cdot 3$ years after surgery (range $0 \cdot 2-10$ years). Based on the results of the latest available postoperative echocardiogram, patients were divided into two groups: those with normal postoperative EDD (EDD $\leqslant 55 \mathrm{~mm})(n=23)$ (median EDD $49 \mathrm{~mm}$, range 40-55) and those with a postoperative EDD $>55 \mathrm{~mm}$ (median 65, range 56 $100, n=11$ ).

Statistical analysis was performed with a Student $t$-test for paired or unpaired data, linear regression analysis and chi or square test, when appropriate. To compare the value of different tests to predict the postoperative persistence of left ventricular enlargement, we also utilized the receiver-operator characteristic (ROC) curves, similarly to previous studies. ${ }^{[4]}$ Results are expressed as median and range, unless otherwise specified.

\section{Results}

CLINICAL COURSE

Patients were followed-up during a median period of 5 years (range 1-10.4 years). Two patients died, one from congestive heart failure and one from a non cardiac disease. The patient who died from heart failure was preoperatively in functional class III, had the largest echocardiographic left ventricular dimensions (EDD $100 \mathrm{~mm}$, ESD $85 \mathrm{~mm}$, FS 15\%, CSA $42 \mathrm{~cm}^{2}, \mathrm{R} / \mathrm{Th}$ ratio $4 \cdot 1$ ), an angiographic ejection fraction of $24 \%$ and a RSV/EDV ratio of $0 \cdot 13$. In this patient, despite an uncomplicated valve replacement, symptoms and left ventricular dimensions did not decrease after surgery. The other patients were seven in class II and 25 in class I.

\section{ECHOCARDIOGRAPHIC RESULTS}

Left ventricular dimensions decreased substantially after valve replacement as summarized in Table 1.

Table I Pre-and postoperative echocardiographic results (median $3 \cdot 3$ years, range $0 \cdot 2-10$ years) after surgery in 34 patients

Pre-operative Postoperative $P$ value

$\begin{array}{lccccc}\text { LV EDD, }(\mathrm{mm}) & 72 & (58-100) & 52 & (40-100) & 0 \cdot 001 \\ \text { LV ESD, }(\mathrm{mm}) & 52 & (32-85) & 35 & (22-90) & 0.001 \\ \text { LV FS, }(\%) & 30 & (14-45) & 30 & (10-49) & \text { NS } \\ \text { LV CSA, }\left(\mathrm{cm}^{2}\right) & 28 & (21-47) & 22 & (14-34) & 0.001 \\ \text { LV R/Th } & 3.5(2 \cdot 1-4 \cdot 4) & 2 \cdot 4(1 \cdot 3-5 \cdot 0) & 0.001\end{array}$

PREDICTION OF PERSISTENT POSTOPERATIVE LEFT VENTRICULAR ENLARGEMENT FROM PREOPERATIVE CLINICAL, CATHETERIZATION AND

ECHOCARDIOGRAPHIC DATA

Patients were divided in two groups according to the latest echocardiographic results during follow-up: 23 patients had normal left ventricular EDD (median $49 \mathrm{~mm}$, range 40-55) and 11 had a persistent left ventricular enlargement (median EDD $65 \mathrm{~mm}$, range 56-100). The interval between surgery and the latest post-operative echocardiogram was not significantly different in the two groups (median $2 \cdot 2$ years, range $0 \cdot 2-8 \cdot 8$ in patient with normal EDD and 3.5 years, 
Table 2 Preoperative data in 34 patients with normal postoperative EDD $(n=23$, group I) or persistent left ventricular enlargement $(n=11$, group 2)

$$
\text { Group } 1 \quad \text { Group } 2 \quad P \text { value }
$$

\begin{tabular}{|c|c|c|c|c|c|}
\hline \multicolumn{6}{|l|}{ Clinical data } \\
\hline Age, (years) & 43 & $(17-73)$ & 55 & $(35-68)$ & $0 \cdot 04$ \\
\hline Males, & 11 & $(48 \%)$ & 10 & $(90 \%)$ & $0 \cdot 02$ \\
\hline NYHA functional class $>$ II & 13 & $(57 \%)$ & 4 & $(36 \%)$ & NS \\
\hline Valve size (only for Björk-Shiley pros.) & 23 & $(21-27)$ & 27 & $(25-29)$ & NS \\
\hline \multicolumn{6}{|l|}{ Echocardiographic data } \\
\hline LV EDD, $(\mathrm{mm})$ & 72 & $(58-90)$ & 77 & $(65-100)$ & NS \\
\hline LV ESD, $(\mathrm{mm})$ & 50 & $(32-68)$ & 55 & $(48-85)$ & $0 \cdot 05$ \\
\hline LV FS, $(\%)$ & 31 & $\left(\begin{array}{ll}14 & 45\end{array}\right)$ & 26 & $(15-38)$ & NS \\
\hline LV CSA, $\left(\mathrm{cm}^{2}\right)$ & 27 & $(21-47)$ & 28 & $(25-41)$ & NS \\
\hline $\mathrm{LV} \mathrm{R} / \mathrm{Th}$ & $3 \cdot 5$ & $(2.4-4.2)$ & $3 \cdot 6$ & $(2.1-4.4)$ & NS \\
\hline \multicolumn{6}{|l|}{ Cardiac catheterization data } \\
\hline LV end diastolic pressure, $(\mathrm{mmHg})$ & 20 & $(6-50)$ & 21 & $(10-38)$ & NS \\
\hline Cardiac index, $\left(1 \mathrm{~min}^{-1} / \mathrm{m}^{2}\right)$ & $3 \cdot 1$ & $(2 \cdot 2-4 \cdot 5)$ & $3 \cdot 1$ & $(2 \cdot 3-5 \cdot 8)$ & NS \\
\hline EDVI, $\left(\mathrm{ml} \mathrm{m}^{-2}\right)$ & 235 & $(128-509)$ & 261 & $(113-360)$ & NS \\
\hline ESVI, $\left(\mathrm{ml} \mathrm{m}^{-2}\right)$ & 129 & $(57-340)$ & 159 & $(47-258)$ & NS \\
\hline $\mathrm{EF},(\%)$ & 50 & $(32-66)$ & 42 & $(24-60)$ & 0.01 \\
\hline Mass index, $\left(\mathrm{g} \mathrm{cm}^{-2}\right)$ & 470 & $(117-470)$ & 467 & $(119-467)$ & NS \\
\hline RSVI, $\left(\mathrm{ml} \mathrm{m}^{-2}\right)$ & 89 & $(35-217)$ & 55 & $(32-123)$ & 0.06 \\
\hline Reg. fraction, $(\%)$ & 67 & $(45-78)$ & 57 & $(41-82)$ & 0.06 \\
\hline RSV/EDV & \multicolumn{2}{|c|}{$0.32(0.24-0.46)$} & \multicolumn{2}{|c|}{$0.25(0.13-0.37)$} & 0.005 \\
\hline
\end{tabular}

range $0 \cdot 2-10$ in the other group). During follow-up, 19 patients with normal postoperative EDD were in functional class I and 4 in class II, while patients with left ventricular enlargement were 6 in class I, 3 in class II and 2 died. The duration of clinical followup in the two groups was comparable (respectively 4.2 years, range $1 \cdot 6-10.4$ and 6 years, range $1-10$ years). Preoperative data in patients with normal and abnormal postoperative LV/EDD are summarized in Table 2. From these data, it appears that the RSV/ EDV ratio and ejection fraction were the variables which showed the greatest difference between the two groups, being lower in patients with persistent postoperative left ventricular enlargement. In Fig. 1 is represented the frequency distribution of the RSV/ EDV ratio in patients with normal and abnormal postoperative left ventricular dimensions. In Figs. 2 and 3 are represented the ROC curves indicating the sensitivity and specificity of some echocardiographic and cardiac catheterization derived variables. The results show that the RSV/EDV has the highest predictive accuracy during almost the whole range of measurements compared with echocardiographic and ventriculographic variables. In Table 3 is summarized

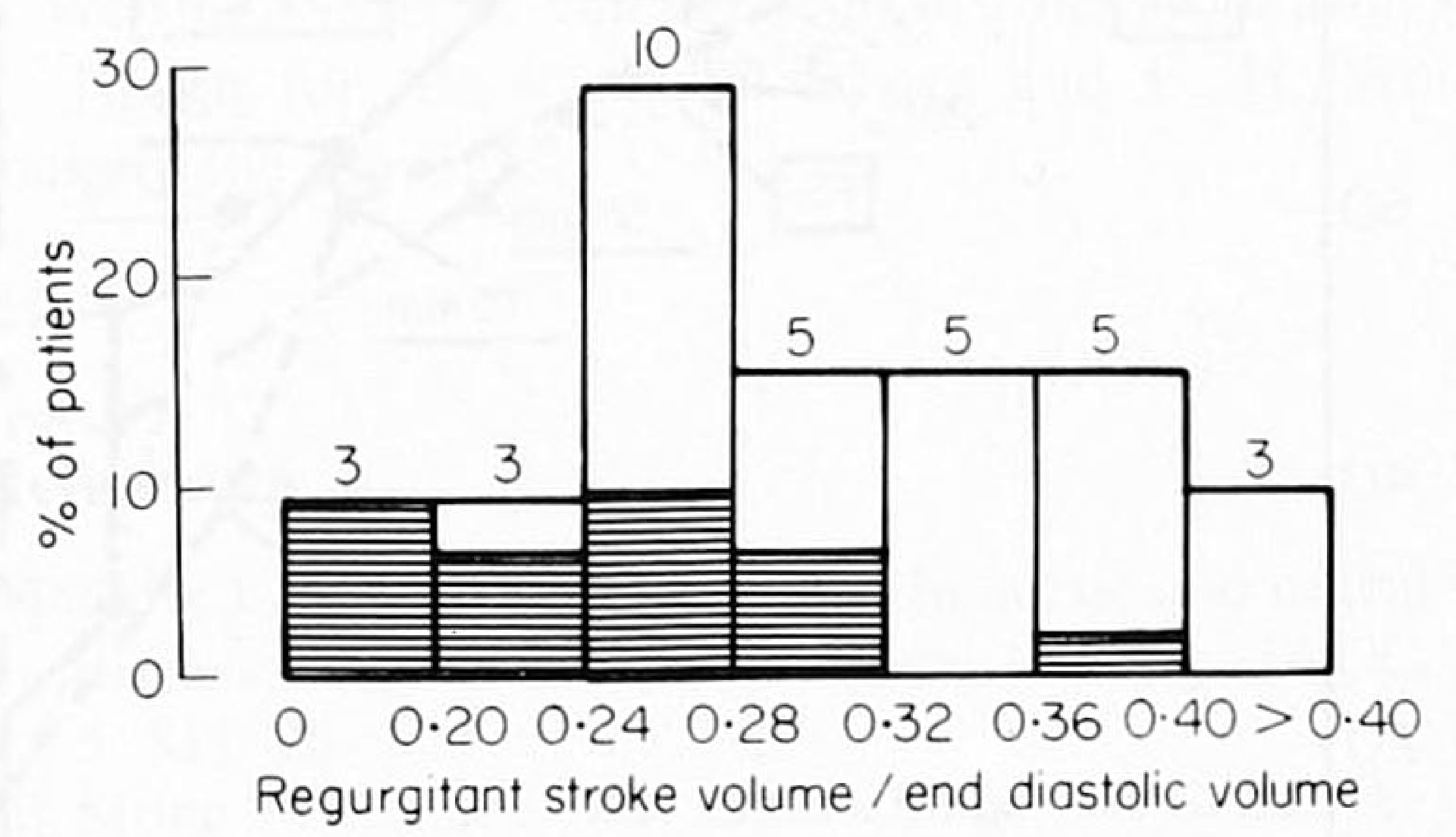

Figure I Frequency distribution of RSV/EDV ratio. $\square$, normal postoperative EDD, $n=23$; 目, abnormal postoperative EDD, $n=11$.

the predictive accuracy of preoperative echocardiographic and cardiac catheterization results. The predictive accuracy is expressed as percentage and $95 \%$ confidence limits of specificity, sensitivity, predictive value positive and negative. The large $95 \%$ confidence limits are due to the small number of patients included in the study. The cut-off points of each variable was selected from visual inspection of the ROC curves and defined as the points with the highest sensitivity and specificity. 
Table 3 Predictive accuracy, in percentage with 95\% confidence limits of some preoperative echocardiographic and cardiac catheterization data for the persistence of left ventricular enlargement after valve replacement.

\begin{tabular}{lllll}
\hline & sensitivity & specificity & PV pos & PV neg \\
\hline Echocardiography & & & & \\
EDD $\geqslant 75 \mathrm{~mm}$ & $73(39-94)$ & $61(39-80)$ & $47(23-72)$ & $18(4-43)$ \\
ESD $\geqslant 55 \mathrm{~mm}$ & $64(31-89)$ & $61(39-80)$ & $43(20-70)$ & $22(6-48)$ \\
FS $\leqslant 30 \%$ & $82(48-98)$ & $61(39-80)$ & $50(26-74)$ & $13(2-38)$ \\
& & & & \\
Cardiac catheterization & & & & \\
EDVI $\geqslant 275 \mathrm{ml} \mathrm{m} \mathrm{m}^{-2}$ & $64(31-89)$ & $52(31-73)$ & $39(17-64)$ & $25(7-52)$ \\
ESVI $\geqslant 150 \mathrm{ml} \mathrm{m}{ }^{-2}$ & $55(23-85)$ & $70(47-87)$ & $46(19-75)$ & $24(8-47)$ \\
EF $<45 \%$ & $73(39-94)$ & $78(56-93)$ & $62(32-86)$ & $14(3-36)$ \\
RSV $/$ EDV $\leqslant 0 \cdot 28$ & $73(39-94)$ & $65(43-84)$ & $50(25-75)$ & $17(4-41)$ \\
RSV $/$ EDV $<0 \cdot 25 *$ & $71(29-96)$ & $89(65-99)$ & $71(29-96)$ & $22(6-48)$ \\
& & & & \\
\hline
\end{tabular}

PV pos, predictive value of a positive test (number of patients with a positive test correctly predicted/all patients with a positive test $\times 100$ ); PV neg, $100-$ (number of patients with a negative test/all patients with a negative test $\times 100)$.

* Data from Levine and Gaasch. ${ }^{[7]}$

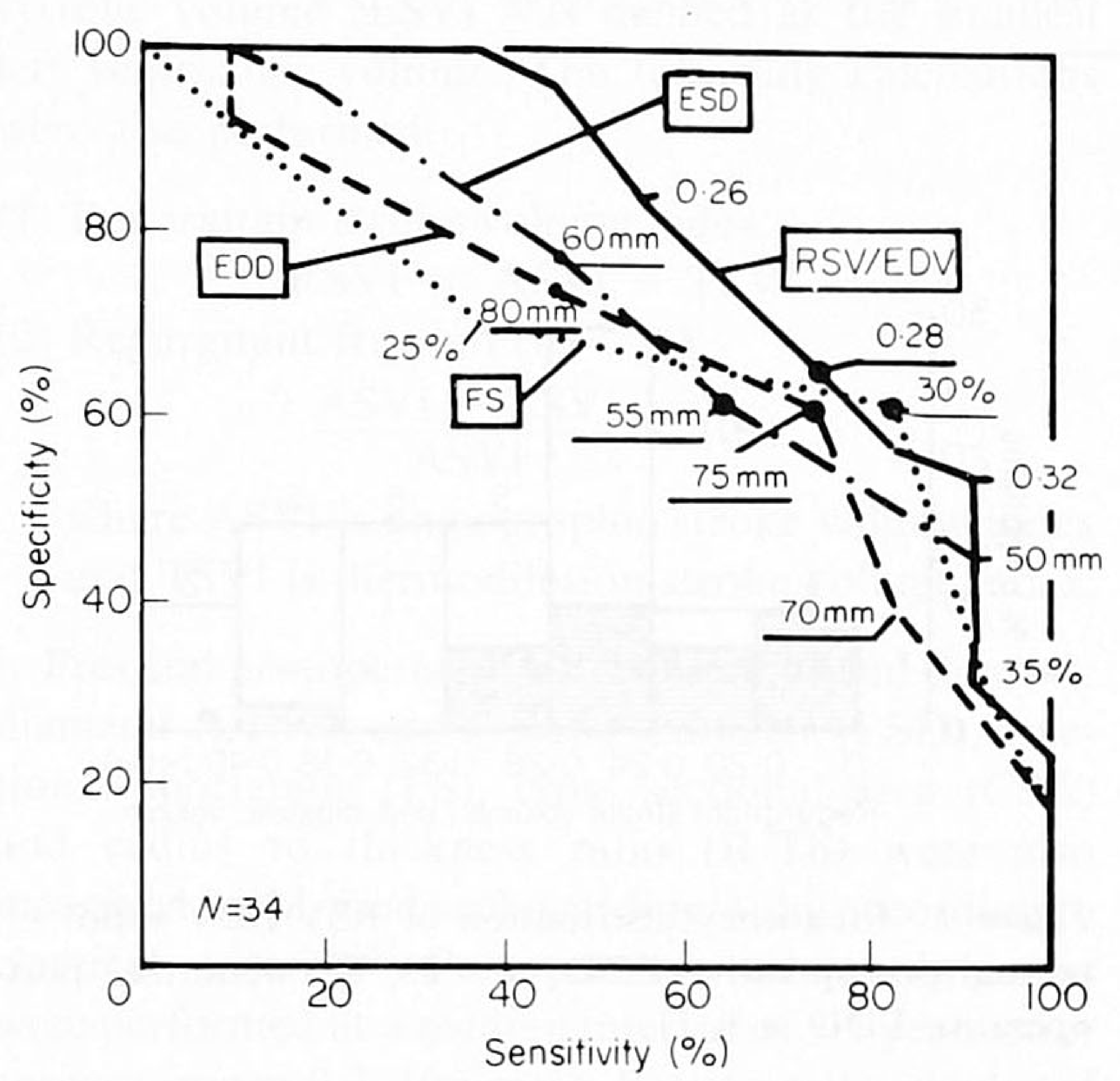

Figure 2 Receiver operator characteristics (ROC) curves indicating specificity (percent of patients with normal postoperative left ventricular dimensions after surgery correctly predicted) versus sensitivity (percent of patients with abnormal postoperative left ventricular dimensions after surgery correctly predicted) of preoperative echocardiographic variables and RSV/EDV ratio. The highest sensitivity and specificity during the whole range of measurement is provided by RSV/EDV ratio and its best cut-off point (that is the point with the highest sensitivity and specificity) corresponded to a ratio of $0 \cdot 28$.

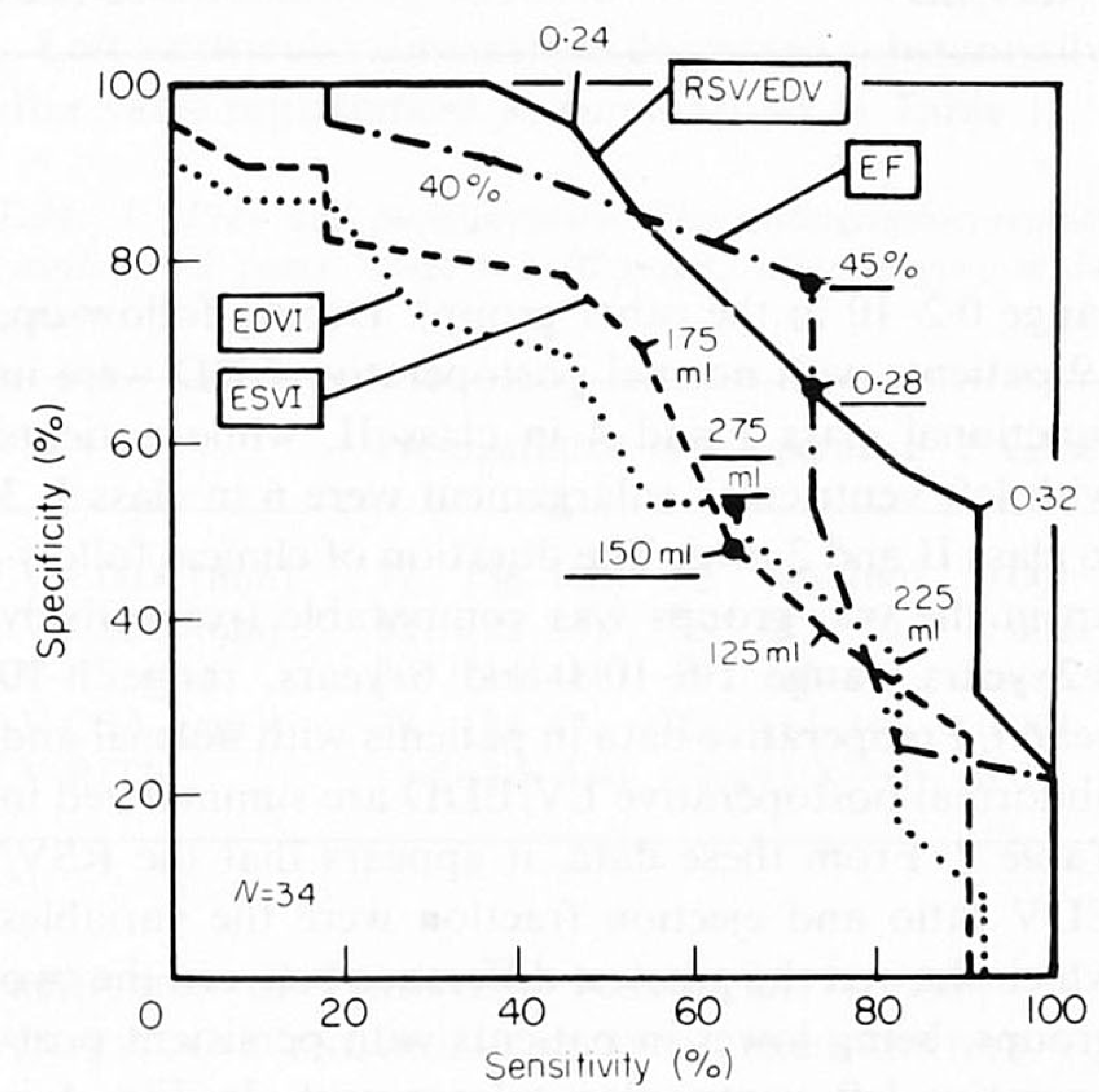

Figure 3 ROC curves of preoperative catheterization derived data to predict postoperative left ventricular dimensions. The predictive accuracy of RSV/EDV ratio was higher than that of ESVI and EDVI and was superior to EF during almost the whole range of measurements. 


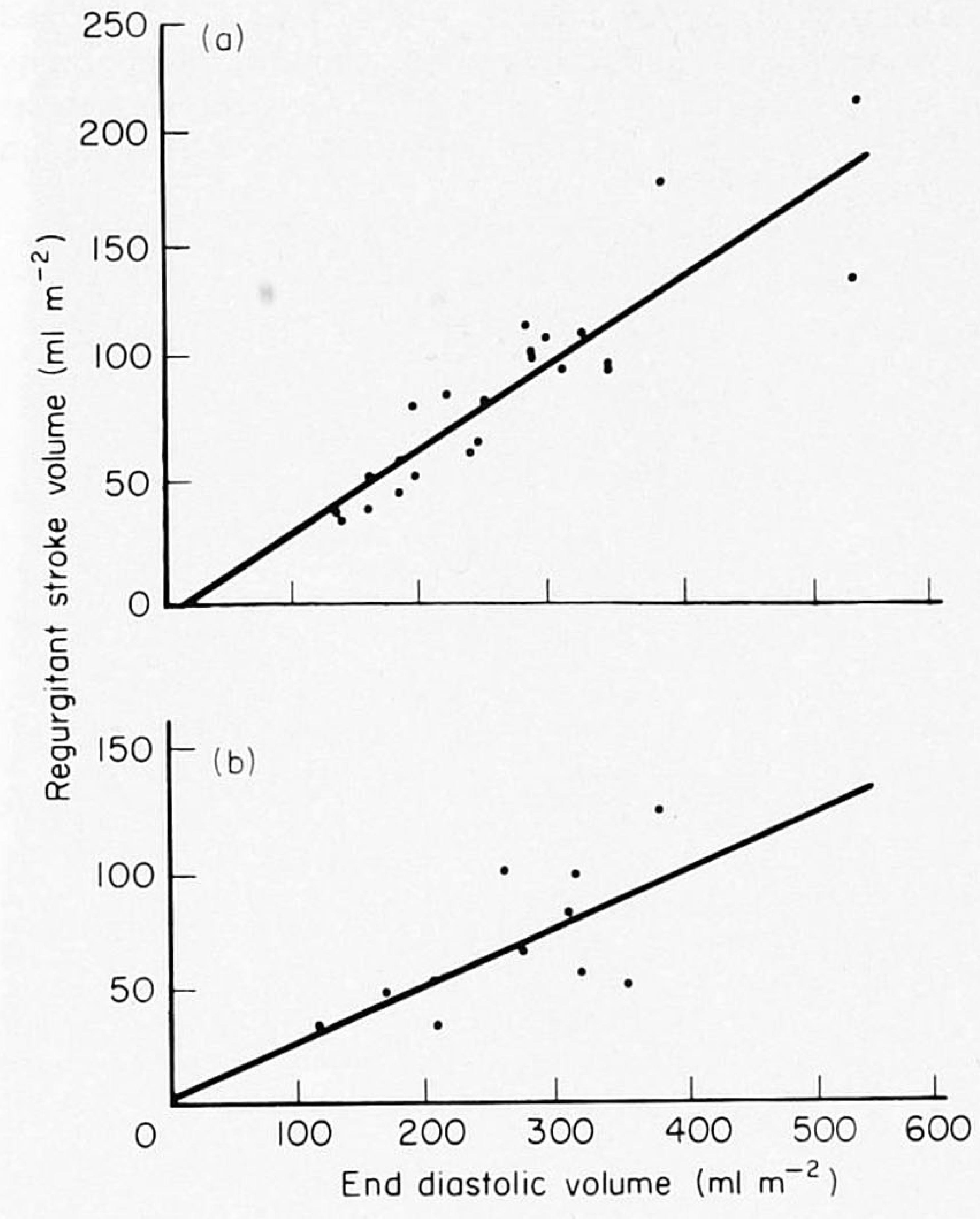

Figure 4 Regurgitant volume versus left ventricular end diastolic volume in (a) 23 patients with normal postoperative left ventricular dimension $(r=0.90, \quad$ SEE $=19$, $n=23$ ) and in (b) 11 patients with persistent postoperative left ventricular enlargement $(r=0.67, \mathrm{SEE}=23, n=11)$.

\section{Discussion}

In the present study we confirmed that the regurgitant stroke volume to end diastolic volume ratio is an important determinant of the regression of left ventricular dimensions after uncomplicated valve replacement for aortic insufficiency.

Our data were very similar to those originally reported by Levine and Gaasch ${ }^{[7]}$ since they found that the 'best' cut-off point to predict a normalization of left ventricular dimensions after surgery was a RSV/EDV ratio of 0.24 , compared with 0.28 in our study. In our study, the left ventricular end-diastolic volume and regurgitant stroke volume were positively correlated in patients with normal as well as in patients with abnormal postoperative left ventricular dimensions (Fig. 4). The correlation was better in patients with good surgical response, and the regression line was steeper in these patients. This indicates that preoperatively, patients with good surgical results had smaller left ventricular dimensions with similar amount of regurgitation, when compared to patients with persistent left ventricular enlargement after valve replacement.
Based on preoperative RSV/EDV ratio, a 'high risk' and a 'low risk' groups could be identified. Postoperative EDD remained abnormal after surgery in 8 out of 16 patients $(50 \%)$ with a RSV/EDV ratio $\leqslant 0 \cdot 28$, while EDD persisted enlarged in only 3 out of 18 patients $(17 \%)$ with a preoperative RSV/EDV greater than $0 \cdot 28$ (Fig. 1, Table 3 ).

Our data further suggest that the predictive value of the RSV/EDV ratio is slightly better or comparable to that of most usual echocardiographic and cardiac catheterization parameters (Figs. 2, 3 and Table 3). The small number of patients included in this study, however, does not allow to conclude that the $\mathrm{RSV} / \mathrm{EDV}$ ratio provides information independent from other parameters.

We acknowledge the limitation of the study due to the selection of patients and to the use of multiple techniques for the measurement of the regurgitation and left ventricular volumes, and for the assessment of follow-up results. However, the confirmation of previous data by Levine and Gaasch ${ }^{[7]}$ suggest that this is a valid research direction and that the RSV/ EDV ratio deserves a place in the evaluation of patients with aortic insufficiency. The measurements will be of more clinical relevance if non invasive methods will provide accurate and reproducible results as recently suggested. ${ }^{[9]}$

We thank Mrs T. van der Kolk for her secretarial work, J. Tijssen for the statistical advice and F. H. Wolf for making the drawings.

\section{References}

[1] Ross J Jr. Afterload mismatch in aortic and mitral valve disease: implications for surgical therapy. JACC 1985; 5: 81126

[2] Stone PH, Clark RD, Goldschlager N, Selzer A, Cohn K. Determinants of prognosis of patients with aortic regurgitation who undergo aortic valve replacement. JACC 1984: 3: 1118 26.

[3] Daniel WG. Hood WP, Siart A el al. Chronic aortic regurgitation: assessment of the prognostic value of preoperative left ventricular end-systolic dimension and fractional shortening. Circulation 1985; 4: 66980.

[4] Fioretti P, Roelandt J, Sclavo M et al. Postoperative regression of left ventricular dimensions in aortic insufficiency: a long term echocardiographic study. JACC 1985; 5: 856 -61.

[5] Bonow RO, Rosing DR, McIntosh CL et al. The natural history of asymptomatic patients with aortic regurgitation and normal left ventricular function. Circulation 1983; 68: 509 17.

[6] Bonow RO, Rosing DR, Kent KM, Epstein SE. Timing of operation for chronic aortic regurgitation. Am J Cardiol 1982; 50: 32536 . 
[7] Levine HJ, Gaasch WH. Ratio of regurgitant volume to end diastolic volume: a major determinant of ventricular response to surgical correction of chronic volume overload. Am J Cardiol 1983; 52: 406-10.

[8] Dodge HT. Determination of left ventricular volume and mass. Rad CL North Am 1971: 9: 459-67.
[9] Klepzig H, Stanake R, Nickelsen T et al. Combined first pass and equilibrium radionuclide ventriculography and comparison with left ventricular/right ventricular stroke count ratio in mitral and aortic regurgitation. Am J Cardiol 1985; 55: 1048-53. 